\section{CÂNCER INFANTIL: PERCEPÇÕES DE CUIDADORAS SOBRE ALIMENTAÇÃO, DINÂMICA FAMILIAR E EMOCIONAL}
Childhood cancer: caregivers' perception regarding nutrition, family dynamics and emotions
Cáncer infantil: percepciones de cuidadoras sobre alimentación, dinámica familiar y emocional

\section{RESUMO}

Objetivo: Conhecer a percepção de cuidadoras sobre alimentação, dinâmica familiar e emocional de crianças e adolescentes com câncer. Métodos: Pesquisa descritiva, com abordagem qualitativa das informações, realizada entre março e maio de $2013 \mathrm{em}$ um centro pediátrico de referência no diagnóstico e tratamento do câncer infantojuvenil, em Fortaleza/ CE. Foram selecionadas, por amostragem não probabilística, aleatória e por conveniência, 24 cuidadoras de crianças com câncer. Coletaram-se informações por entrevista semiestruturada e grupo focal; posteriormente, realizou-se a análise de conteúdo. Resultados: Definiram-se as seguintes categorias: "Dinâmica familiar e suas implicações emocionais", "Preocupação com a alimentação saudável" e "Mitos alimentares". Os conteúdos expõem cuidadoras preocupadas com as mudanças na dinâmica familiar devido à sua ausência constante do lar para dedicarem-se à criança doente. O principal problema enfrentado foi a recusa alimentar da criança doente, pois dele emerge a alimentação como ato de amor, cuidado e cura. As mães referiram aspectos culturais que interferem na organização da dieta de sua família, especificamente os mitos. Conclusão: Na percepção das mães cuidadoras entrevistadas, existem importantes repercussões do diagnóstico de câncer que geram modificações na alimentação, dinâmica familiar e emocional, demandando adaptação da criança doente e de sua família.

Descritores: Câncer; Cuidado da Criança; Alimentação.

\section{ABSTRACT}

Objective: To know caregivers' perception regarding nutrition, family dynamics and emotions of children and adolescents with cancer. Methods: Qualitative descriptive research conducted between March and May 2013 in a reference pediatric center for diagnosis and treatment of cancer in infants and young children in Fortaleza, Ceará. The nonprobability, random and convenience sample included 24 caregivers of children with cancer. Information was collected using semi-structured interviews and focus group, and was later submitted to content analysis. Results: The following categories were defined: "Family dynamics and its emotional implications", "Concern about healthy eating" and "Food myths". The content reveals caregivers concerned about changes in family dynamics due to the constant absence from home to devote to the sick child. The main problem faced was the sick child's refusal to eat, and nutrition emerged as an act of love, care and cure. Mothers reported cultural aspects that interfere in the planning of their family's diet, particularly the myths. Conclusion: From the point of view of the caregiving mothers interviewed, the cancer diagnosis has important consequences that generate changes in the nutrition, family dynamics and emotions, which require the adaptation of the sick child and the family.

Descriptors: Cancer; Child Care; Feeding.
Artigo Original

Mariana Rebouças de Oliveira ${ }^{(1)}$

Rute Matos Dourado Esteves

Justa $^{(1)}$

Marilene Calderaro da Silva

Munguba $^{(1)}$

Larissa da Silva Albuquerque ${ }^{(1)}$

Maria Albertina Rocha

Diógenes $^{(1)}$

Sara Maria Moreira Lima

Verde $^{(1)}$

1) Universidade de Fortaleza - UNIFOR Fortaleza (CE) - Brasil
Recebido em: 10/06/2015 Revisado em: 15/10/2015 Aceito em: 09/11/2015 


\section{RESUMEN}

Objetivo: Conocer la percepción de cuidadoras sobre la alimentación, la dinámica familiar y emocional de niños y adolescentes con cáncer. Métodos: Investigación descriptiva de abordaje cualitativa de las informaciones realizada entre marzo y mayo de 2013 en un centro de pediatría de referencia en el diagnóstico y tratamiento del cáncer infanto-juvenil de Fortaleza, Ceará. Fueron elegidas 24 cuidadoras de niños con cáncer a través de un muestreo no probabilístico, aleatorio y por conveniencia. Se recogieron informaciones a través de entrevista semiestruturada y grupo focal; a posteriori se realizó el análisis de contenido. Resultados: Se definieron las siguientes categorías: "Dinámica familiar y sus implicaciones emocionales", "Preocupación con la alimentación saludable" y "Mitos alimentarios". Los contenidos muestran cuidadoras preocupadas con los cambios de la dinámica familiar debido la ausencia constante del hogar para dedicarse al niño enfermo. El principal problema afrontado ha sido la recusa alimentaria del niño enfermo pues de él emerge la alimentación como un acto de amor, cuidado y cura. Las madres refirieron los aspectos culturales que interfieren en la organización de la dieta de su familia, en especial los mitos. Conclusión: En la percepción de las madres cuidadoras entrevistadas hay importantes repercusiones del diagnóstico de cáncer que generan modificaciones de la alimentación, la dinámica familiar y emocional necesitando adaptación del niño enfermo y su familia.

Descriptores: Neoplasias; Cuidado del Niño; Alimentación.

\section{INTRODUÇÃO}

A Promoção da Saúde se constitui numa das estratégias do setor saúde com o objetivo de embasar ações para influenciar os determinantes e condicionantes da saúde da população, mediante o empoderamento das pessoas, assim como de grupos, organizações e da comunidade para serem atores nesse processo, na perspectiva intersetorial ${ }^{(1)}$.

Ao refletir sobre o alcance das ações voltadas à Promoção da Saúde no contexto do câncer infantojuvenil, identifica-se a necessidade de compreensão das repercussões dessa doença na vida da pessoa e de sua família, e de como, a partir do empoderamento, as ações intersetoriais de apoio podem ser desenvolvidas.

Nesse contexto, é imprescindível entender que o câncer infantojuvenil é raro e representa, no Brasil, 3\% de todos os tumores malignos diagnosticados, sendo a primeira causa de morte por doença nessa faixa etária, superado apenas por acidentes. São estimados para 2015 mais de 11 mil novos casos de câncer em crianças e adolescentes até os $19 \operatorname{anos}^{(2)}$.

O paciente oncológico pediátrico é considerado, desde o diagnóstico, um risco nutricional, tanto pelas alterações metabólicas que a neoplasia provoca como pela própria terapia antineoplásica. É também mais suscetível ao desenvolvimento de deficiências alimentares diretamente associadas à piora na qualidade de vida e resposta ao tratamento, levando ao aumento da morbimortalidade ${ }^{(3)}$.

Trata-se, portanto, de uma doença grave e debilitante, que no âmbito dos cuidados dedicados ao paciente, provoca, além da depleção física, transformações na dinâmica familiar, envolvendo aspectos psicossociais e financeiros, causando transtornos não só pela patologia, mas também pela sobrecarga aos cuidadores, e gerando desequilíbrio no convívio social e familiar ${ }^{(4)}$.

A família se constitui em "[...] uma instituição de união afetiva, de comunhão diária das atividades e compartilhamento das situações na qual seus membros são responsáveis pelo cuidado mútuo, no seu cotidiano"(5). Esse aspecto é considerado pelos paradigmas atuais de assistência à criança, que preconizam a presença e participação dos pais na assistência nas unidades hospitalares ${ }^{(6)}$. No contexto hospitalar, a parceria da família e da criança com a equipe de saúde é permeada de saberes de todos os envolvidos no processo, o que pode contribuir para o empoderamento da família nesse cuidado ${ }^{(7)}$. Em geral, é a mãe que personifica a presença da família nessa perspectiva do cuidado, o que favorece a verbalização de sua percepção sobre esse processo.

Compreender o significado da alimentação para cuidadores dos pacientes em tratamento antineoplásico, suas percepções sobre a afetividade envolvida nesse cuidar, bem como as modificações familiares em função do diagnóstico de câncer pode contribuir para a prestação de uma assistência em saúde mais qualificada pelos profissionais que trabalham com oncologia pediátrica, em especial, o nutricionista.

O objetivo deste estudo foi conhecer a percepção de cuidadoras sobre alimentação, dinâmica familiar e emocional de crianças e adolescentes com câncer.

\section{MÉTODOS}

Trata-se de um estudo descritivo, com abordagem qualitativa, das informações que buscam, através da palavra, as percepções dos seres humanos pertencentes a grupos particulares acerca de determinado processo social, propiciando, assim, a criação de novos $\operatorname{conceitos}^{(8)}$.

A pesquisa ocorreu entre março e maio de 2013, em um centro pediátrico referência no diagnóstico e tratamento do câncer infantojuvenil, localizado na cidade de Fortaleza/ CE.

Definiu-se a seleção dos sujeitos por amostragem não probabilística $^{(9)}$, aleatória, por conveniência, segundo a disponibilidade de participação. Consideraram-se elegíveis 
os cuidadores de crianças e adolescentes em tratamento oncológico há pelo menos um mês no referido centro. Não foram incluídos os cuidadores de pacientes atendidos nos ambulatórios de diagnóstico precoce (pré-tratamento) e de seguimento (pós-tratamento).

O número de participantes ocorreu por saturação teórica da amostra ${ }^{(10)}$, que ocorre quando os dados, na visão do pesquisador, começam a apresentar repetição. Assim, participaram do estudo 24 cuidadores de crianças e adolescentes em tratamento de câncer no referido hospital.

A coleta de informações deu-se por uso da técnica do grupo focal (GF), a qual promove o estímulo da construção de diálogos entre os participantes diante de um tema específico e de perguntas norteadoras ${ }^{(11)}$. Realizaram-se 3 GF, sendo cada um deles constituído de um moderador (a pesquisadora), um colaborador (observador) e, em média, oito participantes. Para sua operacionalização, empregaramse os roteiros de GF e de observação, definindo-se um intervalo de uma semana entre eles, a fim de assegurar a heterogeneidade da amostra. Os GF tiveram duração média de 40 minutos e ocorreram em sala de espera de fácil acesso, em horário de pouco movimento, assegurando, assim, o silêncio e a tranquilidade necessários ao processo.

Ao início de cada GF, realizou-se a caracterização dos dados de identificação dos cuidadores mediante entrevistas semiestruturadas realizadas pela pesquisadora, contendo as variáveis sexo, idade, naturalidade, endereço, profissão, nível de escolaridade e renda familiar, sendo essas informações registradas em instrumento estruturado para o presente estudo.

Efetuou-se a adaptação dos GF compostos por seis perguntas norteadoras. O que mudou na sua família após o adoecimento do seu filho? Você acha que a alimentação pode interferir no tratamento do seu filho? Depois que seu filho iniciou o tratamento, quais as suas principais preocupações em relação à alimentação dele? Após o tratamento, mudou algo na alimentação do seu filho? Quais as suas principais preocupações em relação à alimentação durante o tratamento? Quais suas principais dúvidas em relação à alimentação de seu filho? As falas dos participantes foram gravadas com smartphone, mediante autorização prévia, e suas atitudes, registradas pelo observador.

$\mathrm{Na}$ análise desses documentos, adotou-se a técnica de análise temática de conteúdo, segundo os passos nela previstos $^{(8)}$, evidenciando os significados emergentes no material, de acordo com os objetivos teóricos da pesquisa. A partir do processo analítico e interpretativo encaminhado em relação ao tema em debate neste artigo, identificaramse quatro categorias temáticas: "Dinâmica familiar e suas implicações emocionais", "Preocupação com a alimentação saudável", "Mitos alimentares" e "Dificuldades na alimentação".
O projeto teve a sua aprovação pelos Comitês de Ética em Pesquisa da Universidade de Fortaleza (UNIFOR) (parecer $n^{\circ}$. 189.263) e do Hospital Infantil Albert Sabin (HIAS) (parecer $n^{\circ}$. 215.264), seguindo a Resolução do Conselho Nacional de Saúde n ${ }^{\circ}$.466/2012. Os participantes assinaram o Termo de Consentimento Livre e Esclarecido e a autorização prévia para gravação, tendo assegurada a garantia de sigilo do material obtido. Ressalta-se que, para manter o anonimato das participantes, estas foram denominadas de entrevistadas pela letra E: E1, E2, E3... E assim sucessivamente.

\section{RESULTADOS E DISCUSSÃO}

\section{Caracterização dos participantes}

Participaram do estudo 24 mulheres, todas mães e cuidadoras de crianças e adolescentes internados na enfermaria e na Unidade de Tratamento Intensivo (UTI), das quais 15 eram domésticas, 3 do lar, 2 agricultoras, 1 costureira, 1 pintora, 1 auxiliar de serviços gerais e 1 vendedora autônoma. Todas se afastaram do trabalho após o início do tratamento do filho. Quatorze tinham ensino médio ( 5 delas incompleto e 9 ensino fundamental) e somente 1 concluiu. Uma participante declarou-se não alfabetizada. A renda familiar variou entre menos de um salário mínimo (11 delas) e de 1 a 2 salários mínimos (13 delas). Destacase a prevalência da baixa renda e baixa escolaridade das cuidadoras, associadas à necessidade de interromper sua atividade laboral em função dos cuidados com o filho.

A aproximação com as cuidadoras levou à compreensão de que o enfrentamento da doença envolve uma série de aspectos subjetivos e gera uma cadeia de conflitos emocionais, com inúmeras repercussões na organização da unidade familiar. Tais aspectos, por sua vez, encontram-se intrinsecamente correlacionados com o nutrir. Tanto que, apesar de a maioria das perguntas norteadoras dos GF estarem direcionadas às questões alimentares, o assunto família esteve presente em vários momentos da discussão, tornando-se indissociável do tema central deste trabalho.

Durante o processo de análise das informações, definiram-se as seguintes categorias: "Dinâmica familiar e suas implicações emocionais", "Preocupação com a alimentação saudável"; "Mitos alimentares" e "Dificuldades na alimentação".

\section{Dinâmica familiar e suas implicações emocionais}

$\mathrm{O}$ tratamento da criança e do adolescente com câncer representa um grande desafio tanto para a família como para os profissionais de saúde envolvidos no processo. O diagnóstico de uma neoplasia provoca mudanças abruptas na dinâmica familiar do indivíduo, interferindo na rotina de 
vida e gerando um elevado grau de ansiedade por resposta à doença $a^{(7,12)}$.

Durante o tratamento oncológico, os cuidadores são impelidos a se adaptar a uma nova rotina familiar, social e profissional, e a lidar com uma série de eventos estressantes, como medos, expectativas, medicações, internamentos e efeitos colaterais ${ }^{(13)}$. Muitas famílias, em decorrência da sobrecarga de atividades, que é maior na população de baixa renda, acabam vivenciando situações de conflitos entre os parentes ${ }^{(14)}$.

A doença do filho gera um grande sofrimento à mãecuidadora, por ter que se ausentar de casa e do convívio com os outros filhos e pessoas queridas, principalmente durante os períodos de hospitalização, como registrado na fala a seguir:

\section{Mudou tudo. Toda rotina dela mudou. (E1)}

As mães, geralmente, tornam-se cuidadoras primárias; deixam o emprego e o restante da família e centralizam os cuidados na criança doente em detrimento do marido e dos demais filhos. Concentram em si diversas responsabilidades do cotidiano (administração de remédios, intercorrências, decisões, dentre outras). Cria-se, então, uma nova situação de vida, com o pai no papel de provedor e a mãe assumindo as principais responsabilidades relacionadas ao tratamento, conforme se pode perceber neste relato:

Assim que eu soube da doença, deixei tudo, me mudei do interior pra cá com ele. E a gente vive aqui [no hospital]. O marido ficou lá. É difícil... (E2)

Ao analisarem o cotidiano de cuidadores informais de pacientes em quimioterapia, pesquisadores ${ }^{(15,16)}$ encontraram maior prevalência de cuidadores do sexo feminino. Ao que indica, esses achados podem ser justificados pelo fato de a mulher assumir, historicamente, a função de cuidadora da família, principalmente dos filhos ${ }^{(17)}$. Encontrouse realidade análoga entre as mães participantes deste estudo, pois todas deixaram o trabalho e se dedicaram exclusivamente ao tratamento dos filhos, sendo a maioria advinda de municípios do interior do estado, onde haviam deixado seus familiares.

\section{Preocupação com a alimentação saudável}

Para a mãe, o diagnóstico de câncer é uma experiência dolorosa e chocante. Ao receber a confirmação da doença, os familiares vivenciam sentimentos de temor, apreensão e dúvidas em relação aos procedimentos multidisciplinares inerentes ao tratamento ${ }^{(18,19)}$. Algumas falas ilustram essa situação:

No mesmo instante [em] que a doutora me deu o diagnóstico, eu voltei a respirar depois de uma breve parada cardiaca... "Volta que tu é mãe!” (sic). Então, eu falei: "Sim, o que faz mal a ele? O que ele pode comer?". Eu queria saber o que é que eu não podia fazer. (E3)

Assim, a categoria "Preocupação com a alimentação saudável" emerge e evidencia que o tratamento do câncer afeta a alimentação do paciente justo no momento em que ele precisa melhor se alimentar. Desta feita, a nutrição torna-se essencial nos cuidados e na terapêutica. Percebese nas falas das participantes que a preocupação com a alimentação está presente desde o momento do diagnóstico:

Nas primeiras consultas, eu já procurei a nutricionista para me dar o cardápio. E aí, eu sempre sigo. (...) Eu sempre procuro olhar o que é que eu devo dar. (E4)

No mesmo instante [em] que a doutora me deu o diagnóstico (...), falei: "O que faz mal a ele? O que ele pode comer?" (...). Fui logo vendo o seguinte: o que faz bem, o que pode, o que nunca pode e quando pode. (E5)

A vivência de uma doença como o câncer implica em várias adaptações, tanto na vida do doente como de toda a família. Além de reavaliar conceitos pré-existentes e atribuir novos significados à própria vida, a inserção de hábitos até então pouco valorizados é recorrente logo ao diagnóstico, ao início do tratamento e durante todo o processo. A ligação entre alimentos saudáveis e aqueles que fazem bem ficou evidente nas falas das cuidadoras. Além disso, esses alimentos apareciam como aqueles não industrializados. As cuidadoras relataram a alimentação saudável da seguinte maneira:

Comidinha da hora. Comidinha forte. Feijão cozinhado na hora, carne, verdura... Comida de panela, comida saudável. (E6)

Ele sempre se alimentou muito bem em casa, com relação a frutas, legumes, suco... Refrigerante, quando tomava, era só final de semana, e Coca-Cola, não. (E8)

A alimentação correta está relacionada aos alimentos considerados bons para a saúde, como frutas, hortaliças, feijão e carne. Já o alimentar-se bem pressupõe a exclusão de todos os alimentos considerados prejudiciais, os quais, geralmente, são banidos da dieta, com o intuito de prevenir agravos ao estado geral de saúde:

Mudou [a alimentação]. Carne de porco, sardinha, camarão... Essas coisas que dizem que é remoso (sic). Chocolate, macarrãozinho de pacote, que ela sempre foi alucinada por ele... Tirei mais o corante. Tirei a mortadela, que também ela comia muito... Linguiça, manga. (E4)

Ele não consome açúcar; o açúcar dele é o mascavo ou o mel. Tirei literalmente o glúten, coisas assim. Conservante ele não consome, tipo enlatado, salsicha. (E20) 
Em âmbito geral, os cuidadores demonstraram conhecimento sobre alimentação saudável, baseados numa constante dicotomia, em que os alimentos são classificados como bons ou ruins, certos ou errados, saudáveis ou não saudáveis, e, que, por isso, "podem ou não podem" ser consumidos. Essa percepção surge no relato a seguir:

Um diz que beterraba com cenoura não pode. Ai eu perguntei à nutricionista, que disse que não, que pode. A pessoa diz, a gente já fica com medo de dar. Abacaxi não pode, ai a gente fica com medo de dar abacaxi. Uns dizem: "Ah, mulher, isso daqui faz mal", "Ah, mulher, isso daqui é bom, as defesas vão lá em cima", "Ah, mulher, isso daqui não pode não, derruba as defesas". Ai a gente fica assim, em dúvida do que pode e do que não pode. (E18)

Essa divisão dos alimentos em bons e ruins pode ser decorrente do aumento da preocupação em relação ao cumprimento de uma dieta adequada, que causa uma pressão nos indivíduos, levando-os a categorizar os alimentos a partir de seus conhecimentos prévios. Por outro lado, pode simplesmente ter sua origem nas raízes culturais e nos discursos simplistas difundidos pelos profissionais de saúde perante a sociedade, nos quais se preconiza a exclusão de alimentos nocivos ${ }^{(20)}$.

A tendência é que essa preocupação com a alimentação do indivíduo com câncer permaneça mesmo ao término do tratamento, para promover a prevenção da doença e a manutenção da qualidade de vida ${ }^{(19)}$, uma vez que diversas pesquisas ressaltam o efeito protetor que uma alimentação rica em frutas e verduras e pobre em gorduras saturadas teria sobre o surgimento do câncer ${ }^{(21)}$. As cuidadoras questionaram a provável relação entre a má alimentação e o surgimento do câncer:

É porque eu acho, assim, que foi de tanto ele comer "bagulho véi"(sic) que causou a doença. Ele não se alimentava direito, faltava as proteinas que precisava no corpo dele... Aí eu penso assim... [Ele] Só queria comer "bagulho". (E16)

Entretanto, houve relatos contrários a essa ideia:

Eu acho que a alimentação não interfere. Ela tem 1 ano e 6 meses. A alimentaçãozinha dela é só sopa e mingau. (E8)

As causas do câncer na infância ainda não são bem conhecidas. Nessa faixa etária, as neoplasias malignas parecem ser, em sua maioria, de origem embrionária e acometem, geralmente, as células do sistema sanguíneo e dos tecidos de sustentação ${ }^{(22)}$. Portanto, destacam-se esse questionamento entre as cuidadoras e a necessidade de esclarecimentos profissionais que minimizem mais essa preocupação.

\section{Mitos alimentares}

As falas evidenciam que o alimento, apesar de preencher as necessidades fisiológicas, não é ingerido meramente por razões nutricionais. A nutrição está condicionada a diversos fatores, tanto biológicos como sociais e culturais. Historicamente, $\mathrm{o}$ ato de se alimentar encontra-se permeado de crenças e representações que perpassam os anos e convivem em paralelo com os avanços das ciências. Desse modo, os conhecimentos dietéticos tradicionais são transmitidos geração após geração ${ }^{(23)}$.

Ao comentar sobre as mudanças na alimentação do paciente pós-tratamento, percebe-se que estão arraigados às falas das participantes estigmas e mitos relacionados à nutrição:

Uma amiga minha ficou amarela. É por isso que minha irmã me ligou dizendo que a cenoura fazia mal. (...) Ai, ela fica me ligando dizendo que cenoura com beterraba faz mal. É mito. É porque ela deu muita cenoura pro menino e a palma da mão dele ficou amarela. (E12)

Os mitos são histórias que fazem parte da cultura de um povo como forma de explicar fenômenos sem compreensão ${ }^{(23)}$. Os relatos das cuidadoras revelam que, durante o processo saúde-doença, alguns alimentos costumeiramente consumidos e que outrora estavam associados à manutenção da saúde e do bem-estar passam a não ser recomendados em situações de fragilidade do organismo. Para a maioria das participantes deste estudo, alimentos como manga, carne de porco, frutos do mar e alguns tipos de peixes estão associados à piora do quadro de saúde, devendo ser evitados:

A minha come manga. Acho que não é remoso (sic) não, é? (E16).

Observou-se situação semelhante à encontrada neste estudo em pesquisa realizada com mulheres de uma comunidade tradicional do Mato Grosso do $\mathrm{Sul}^{(24)}$ na qual os alimentos ditos fortes - ou que possuem mais "sustância", conforme termo empregado -, como a carne de porco, são associados ao fortalecimento do "organismo saudável" e à recaída do "organismo doente", que não estaria preparado para receber um alimento "forte" e "quente".

Tais "alimentos proibidos" estariam associados à "reima". Assim, percebe-se nas falas da maioria das participantes o temor em relação aos alimentos ditos "reimosos", que seriam prejudiciais ao tratamento:

As coisas reimosas acho que pioram sim. Carne de porco, camarão... (E22)

Agora, essas outras coisas fazem é ajudar.. Verdura, fruta... Não fazem mal. (E24) 
O alimento "reimoso" é aquele que "não é manso" e, por isso, torna-se ofensivo em alguns estados do organismo, podendo ser consumido somente por pessoas em perfeitas condições de saúde. Amplamente disseminada nas regiões Norte, Nordeste e Centro-Oeste do país, a "reima" está relacionada aos efeitos prejudiciais que alimentos causariam sobre o sangue ${ }^{(25)}$.

\section{Dificuldades na alimentação}

Quando se questionou às cuidadoras sobre as mudanças ocorridas na alimentação após o início do tratamento, percebeu-se, no relato dessas mulheres, que a anorexia e a consequente redução da ingestão durante os ciclos quimioterápicos são citadas como principais responsáveis pela alteração no padrão alimentar dos pacientes. Essa referida inapetência, atribuída pela maioria das cuidadoras ao aparecimento de náusea e vômito, é uma das grandes preocupações:

Ele passou a semana internado e na semana seguinte ele não comeu nada. Cinco meses vomitando. Aí fica difícil, ele só tem 3 anos, não entende ainda muita coisa. E se der à força é pior, que ele provoca (vomita) mesmo. Ai é muito difícil. (E14)

De fato, quimioterapia e radioterapia ocasionam uma série de efeitos colaterais de grande repercussão sobre a ingestão alimentar. As alterações metabólicas provocadas pela doença, no entanto, também podem ser responsáveis por tais ocorrências, fato não relatado pelas participantes do estudo. Os agentes quimioterápicos, em especial, induzem ao vômito, à anorexia, à diarreia e à constipação, podendo prejudicar a absorção de nutrientes ${ }^{(3)}$. A presença desses efeitos deixa o organismo debilitado e interfere na ingestão de alimentos, aumentando o risco de comprometimento nutricional e imunológico, promovendo uma piora da qualidade de vida, diminuindo a resposta ao tratamento e afetando de modo negativo o estado geral de saúde do paciente $^{(21)}$.

A dificuldade de se alimentar é uma das mais frequentes queixas apresentadas pelos pais de crianças, inclusive sadias, desde a introdução da alimentação complementar. Costuma suscitar preocupações e angústias em relação ao desenvolvimento infantil.

Apesar de a desnutrição ser um achado frequente entre os pacientes oncológicos e aparecer como uma das principais preocupações dos profissionais de saúde, para as cuidadoras, a maior preocupação é a imunidade da criança:

A gente percebe que as defesas... A fortificação do organismo vai pela alimentação (...). A alimentação fortifica mesmo. Se não se alimentar, fica fraco, fica com febre e as defesas baixam. (E18)
Uma criança bem alimentada tem bem menos chances de cair as defesas (...). Você tem que procurar o tipo de alimento para que não venha descer tanto as defesas dele. Eu acredito que a alimentação é 50\%. (E5)

Observa-se, ainda, em vários relatos, que a redução do consumo de alimentos e do número de refeições é exacerbada nos períodos de hospitalização:

O meu [filho], no primeiro ciclo, quase não se internava. Agora, a própria medicação de recaída é bem mais forte, ai ele para de comer. Ele passou duas semanas sem comer, vomitava, não comia, então a tendência é ficar fraco e a quimioterapia derrubar. (E7)

Essa redução pode estar diretamente relacionada à própria condição fisiológica, aos efeitos colaterais da quimioterapia, às mudanças nos hábitos alimentares e à insatisfação com as refeições ofertadas no internamento ${ }^{(26)}$. Apesar de existir uma preocupação com o estado nutricional do paciente hospitalizado, dá-se pouca atenção à verificação da aceitação da dieta. Nesta pesquisa, não foram identificados relatos que associassem a baixa aceitação da alimentação servida no hospital ao descontentamento com a qualidade da comida servida.

\section{CONSIDERAÇÕES FINAIS}

Ter uma criança ou um adolescente em tratamento oncológico gera grandes mudanças na dinâmica familiar do cuidador, que é impelido a se adaptar a uma nova rotina de cuidados em saúde, deixando em segundo plano seu círculo afetivo e suas relações sociais. O diagnóstico do câncer, para o cuidador, além de ser uma experiência dolorosa, suscita temor e apreensão em relação ao tratamento quimioterápico e à alimentação da criança e do adolescente durante esse período.

A alimentação é vista como parte importante da terapia antineoplásica e essencial para o sucesso no tratamento. Tanto que, ao início deste, a família geralmente modifica antigos hábitos alimentares, excluindo da dieta alimentos industrializados e "reimosos", considerados prejudiciais, aumentando o consumo de alimentos considerados saudáveis, como frutas e verduras. Entretanto, para as cuidadoras, o grande desafio é encontrar meios para contornar a dificuldade de se alimentar do paciente.

Nesse cenário, salienta-se que os cuidadores e o próprio paciente precisam ser rotineiramente orientados pelos profissionais de saúde quanto ao consumo adequado dos alimentos. Esses profissionais têm papel preponderante no tratamento da criança e do adolescente com câncer, devendo estar atentos também às questões subjetivas inerentes a esse processo. 


\section{REFERÊNCIAS}

1. Ministério da Saúde (BR), Secretaria de Vigilância em Saúde, Secretaria de Atenção à Saúde. Política Nacional de Promoção da Saúde: PNPS: revisão da Portaria MS/GM n ${ }^{\circ}$ 687, de 30 de março de 2006. Brasília: Ministério da Saúde; 2015.

2. Instituto Nacional de Câncer (BR). Câncer da criança e adolescente no Brasil: dados dos registros de base populacional e de mortalidade. Rio de Janeiro: Inca; 2008.

3. Bauer J, Jurgens H, Frauhwald MC. Important aspects of nutrition in children with cancer. Adv Nutr. 2011;2(2):67-77.

4. Anjos C, Santo FHE, Carvalho EMMS. Childhood cancer in the family environment: an integrative review. REME Rev Min Enferm. 2015;19(1):227-33.

5. Mantovani MF, Mazza VA, Moreira RC, Silva DI, Jesus JKF, Oliveira VBCA. Representações sociais da família para a equipe da estratégia saúde da família. Rev Enferm UERJ. 2014;22(6):796-800.

6. Melo EMOP, Ferreira PL, Lima RAG, Mello DF. Envolvimento dos pais nos cuidados de saúde de crianças hospitalizadas. Rev Latinoam Enferm (online). 2014;22(3):432-9.

7. Collet N. Sujeitos em interação no cuidado à criança hospitalizada: desafios para a Enfermagem Pediátrica. Rev Bras Enferm. 2012;65(1):7-8.

8. Minayo MCS. O desafio do conhecimento: pesquisa qualitativa em saúde. 12a ed. São Paulo: Hucitec; 2010.

9. Fontanella BJB, Luchesi BM, Saidel MGB, Ricas J, Turato ER, Melo DG. Amostragem em pesquisas qualitativas: proposta de procedimentos para constatar saturação teórica. Cad Saúde Pública. 2011;27(2):38894.

10. Fontanella BJB, Ricas J, Turato ER. Amostragem por saturação em pesquisas qualitativas em saúde: contribuições teóricas. Cad Saúde Pública. 2008; 24(1):17-27.

11. Backes DS, Colomé JS, Erdmann RH, Lunardi VL. Grupo focal com técnica de coleta e análise de dados em pesquisas qualitativas. Mundo Saúde. 2011;35(4):43842.

12. Duarte MLC, Zanini LN, Nedel MNB. O cotidiano dos pais de crianças com câncer hospitalizadas. Rev Gaúch Enferm. 2012;33(3):111-8.

13. Grant $\mathrm{CH}$, Traesel ES. Vivências de cuidadores de crianças e adolescentes com câncer: uma reflexão sobre o apoio psicológico. Disciplinarum Scientia. 2010;11(1):89-108.

14. Fagundes CSO, Silva MF, Silva Junior RF, Barbosa HA. "Senti culpa, muita tristeza e vontade de chorar" - percepções sobre o câncer para mães e cuidadores de crianças em tratamento oncológico. Rev Bionorte. 2015;4(2):48-60.

15. Toledo MO, Ballarim MLGS. O cotidiano de cuidadores informais de pacientes em tratamento quimioterápico. Cad Ter Ocup UFSCar (Impr). 2013;21(1):75-81

16. Amador DD, Gomes IP, Reichert OS, Collet N. Repercussões do câncer infantil para o cuidador família: revisão integrativa. Rev Bras Enferm. 2013;66(2):26770.

17. Ignacio MG, Storti DC, Bennute GRG, Lucia MCS. Aspectos da sobrecarga em cuidadores de pacientes terminais por câncer: revisão de literatura. Psicol Hosp (São Paulo). 2011;9(1):24-46.

18. Santos LF, Marinho KC, Oliveira RR, Siqueira KM, Oliveira LMAC, Peixoto MKAV et al. Ser mãe de criança com câncer: uma investigação fenomenológica. Rev Enferm UERJ. 2011;19(4):626-31.

19. Salci MA, Marcon SS. Após o câncer: uma nova maneira de viver a vida. Rev Rene. 2011;12(2):372-83.

20. Rodrigues EM, Boog MCF. Problematização como estratégia de educação nutricional em adolescentes obesos. Cad Saúde Pública. 2006;22(5):923-93

21. Prado BBF. Influência dos hábitos alimentares de vida no desenvolvimento do câncer. Ciênc Cult (São Paulo). 2014;66(1):21-4.

22. Dal Bosco SM. Terapia nutricional em pediatria. São Paulo: Atheneu; 2010. p. 395.

23. Diez-Garcia RW, Castro IRR. A culinária como objeto de estudo e de intervenção no campo da Alimentação e Nutrição. Ciênc Saúde Coletiva. 2011;16(1):91-8.

24. Woortman AAWK. A comida, a família e a construção do gênero feminino. Dados. 1986;29(1):103-30.

25. Junior LCB, Estácio AG. Tabus alimentares em medicina: uma hipótese para fisiopatologia referente aos alimentos remosos. Rev Assoc Med Bras. 2013;59(3):213-6.

26. Ferreira D, Guimarães TG, Marcadenti A. Aceitação de dietas hospitalares e estado nutricional entre pacientes com câncer. Einstein. 2013;11(1):41-6. 


\section{Endereço do primeiro autor:}

Mariana Rebouças de Oliveira

Universidade de Fortaleza - UNIFOR

Av. Washington Soares, 1321

Bairro: Edson Queiroz

CEP: 60.811-905 - Fortaleza - CE - Brasil

E-mail: mariana_reboucas@hotmail.com

\section{Endereço para correspondência:}

Sara Maria Moreira Lima Verde

Universidade de Fortaleza - UNIFOR

Av. Washington Soares, 1321

Bairro: Edson Queiroz

CEP: 60.811-905 - Fortaleza - CE - Brasil

E-mail: saram@unifor.br 